\title{
Elderly care
}

83 HEAR THE RIGHT STORY AND FINISH UP IN COUNTRY: LEARNING LESSONS FROM ABORIGINAL AUSTRALIANS IMPLEMENTING ADVANCE CARE PLANNING

interviews and focus groups with health and social care workers providing chronic illness and palliative care services in Aboriginal communities. Many of these communities are in remote areas with limited access to health care facilities. Patients and support people have to leave their country (community land) and travel long distances to receive treatment for cancer, chronic kidney disease or other illnesses.

Data analysis indicated the need for conversations and planning for both clinical and the social care. Three major themes arose from the data.

The right story: including the use of language, correct information, avoiding unwanted treatment, and knowing the patient's wishes.

Right people: identifying the decision makers, who should be involved in conversations and who should be told, wider dissemination of the story. Finishing up: importance of spiritual well being, ceremony and ritual, familial ties to country, and people present.

Implementing ACP enabled a cultural group that is often underserved, to be able to make choices about their treatment options and to finish up - to die in country. We conclude that while Aboriginal practices are specific to their culture, many of the subthemes are relevant to patients and families across cultures. Understanding the deeper social issues that underlie ACP conversations and decision making will enhance health and social care workers ability to implement ACP enabling patients and their families to experience appropriate care.

10.1136/bmjspcare-2011-000053.83

R Ray', A Street ${ }^{2}$, J Blackford² ${ }^{1}$ James Cook University; ${ }^{2}$ La Trobe University

As part of a national study evaluating a model of advance care planning (ACP) in community palliative care we conducted 